\title{
University Index
}

Aston University

University of Bath

University of Birmingham

Cardiff University

University of East Anglia

Glasgow University

Imperial College

University of Leicester

Loughborough University

University of Manchester Institute of Science and Technology

University of Oxford

Queen's University Belfast

Sheffield Hallam University

University of Sunderland

Thames Valley University

University of Ulster

University of the West of England

University of York 